\title{
Air Interface of an ATM Radio Access Network
}

\author{
Dietmar Petras
}

Bosch Telecom GmbH

Backnang, Germany

telffax: +49 7191 13-3905/-4603, e-mail: dietmar.petras@pcm.bosch.de

\begin{abstract}
This paper describes system and protocol aspects of the air interface of a fixed ATM Radio Access Network with directional antennas at terminals and sectored antennas at base stations (also called Point-to-Multipoint system). A requirement on the system is that it has to fit transparently into a fixed ATM network. A radio sector of the system is interpreted as a distributed ATM multiplexer. The protocol stack of the air interface implements the statistical multiplexing of ATM cells with a quality of service as in fixed ATM multiplexers with the same link data rate. The multiplexing is controlled by a service strategy that optimizes the resource allocation based on shortterm demands of virtual channels and their negotiated quality of service. The MAC protocol realizes the transmission order of ATM cells given by the service strategy. By this, the protocol stack is able to efficiently support all ATM service categories.
\end{abstract}

\section{Keywords}

Broadband Radio Access Network (BRAN), Point-to-Multipoint System, wireless ATM, quality of service, ATM cell scheduler, MAC protocol, random access

\section{INTRODUCTION}

The development in modern telecommunications is currently determined by the ongoing migration of the worldwide infrastructure to a broadband multimedia network. Multimedia applications require circuit-switched services with guaranteed capacity and transfer delays for interactive voice and video services as well as packet switched services with on demand capacity for the transmission of text, pictures and data. Therefore, multimedia networks are based on the Asynchronous Transfer Mode (ATM), since its connection-oriented packet switched transport allows the connection specific guarantee of capacity and delays. The operator of an ATM network has to install, operate and maintain only one common service integrating network technique instead of the previous multiple service specific networks. Beside this saving in costs, ATM networks are a future proof investment, since their service independent transport in general allows the support of future services.

Another trend in modern telecommunications is given by the success of cellular 


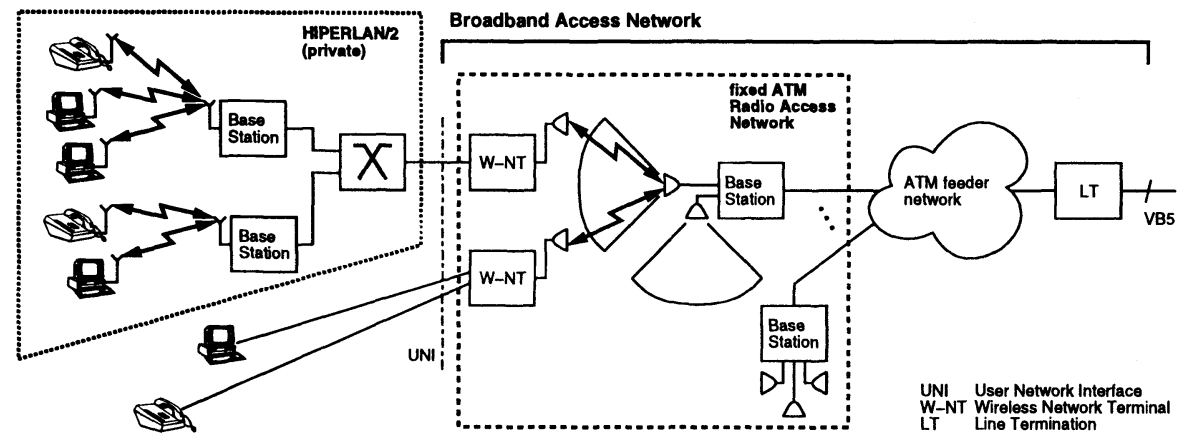

Figure 1 Different applications for wireless ATM systems and architecture of fixed ATM Radio Access Network

mobile radio networks, which serve the users demand for universal accessibility. The current cellular network technique allows the global availability of voice and narrowband data services up to several $10 \mathrm{kbit} / \mathrm{s}$. However, the demand for much higher bandwidths is growing rapidly. But making available data rates of several $1 \mathrm{Mbit} / \mathrm{s}$, as usual at the user access of cable-based multimedia networks, would require a much wider frequency spectrum. This is only available at frequencies higher than $2 \mathrm{GHz}$ where line-of-sight operation is necessary. Thus, a full coverage mobile multimedia network would force an enormous number of base station so that its realization is neither technically nor economically feasible. But there are still two market segments, where ATM radio networks seam to be realistic (Figure 1):

1. ATM-based cordless system or local area radio networks (wireless ATM local area network, W-ATM-LAN) in an unlicensed frequency band with limited mobility in a restricted area (e.g., office building, exhibition area)

2. Broadband fixed radio access (B-FRA) in licensed frequency bands without mobility as replacement of cable-based infrastructure for subscriber access

The second segment is especially stimulated by the current world-wide deregulation in telecommunications, since radio systems allow new operators, which usually don't own a cable-based access network, to gather broad coverage fast and efficiently.

There are a number of research activities in science and industry investigating W-ATM systems (Walke et al., 1996; Radimirsch, 1997; Dinis et al., 1997; AlaLaurila and Awater, 1997; Eng et al., 1995; Raychaudhuri et al., 1997; Porter and Hopper, 1995; Agrawal et al., 1996; Ciotti, 1996). Also international standardization bodies have started W-ATM activities and are planing first technical standards for end of 1998 (BRAN Project, 1997; Wireless ATM Group, 1997; DAVIC, 1997). The ETSI project Broadband Radio Access Networks (BRAN) is dealing with the air interface of two W-ATM systems according to the above two market segments, 
Table 1 Parameters of the ATM Radio Access Network

\begin{tabular}{ll}
\hline frequency band & $3.5,10,24 / 26,28 \mathrm{GHz}$ and higher \\
\hline channel bandwidth & $6 \times 14 \mathrm{MHz}$ duplex @ 3.5GHz \\
& $5 \times 30 \mathrm{MHz}$ duplex @ 10GHz \\
& $\begin{array}{l}18 \times 28 \mathrm{MHz} \text { duplex @ 24/26GHz } \\
\text { block allocation @ 28GHz }\end{array}$ \\
\hline range & $\begin{array}{l}10-15 \mathrm{~km} @ 3.5 \mathrm{GHz} \\
7-10 \mathrm{~km} @ 10 \mathrm{GHz} \\
3-5 \mathrm{~km} @ 24 / 26,28 \mathrm{GHz}\end{array}$ \\
\hline multiplex & frequency division multiplex (FDM) \\
\hline duplex & frequency division duplex (FDD) \\
\hline downstream data rate & 8 - 51 Mbit/s \\
\hline upstream data rate & 2 - 25 Mbit/s \\
\hline medium access & asynchronous multiplex of ATM cells \\
\hline
\end{tabular}

which are called "HIPERLAN type 2" and "HIPERACCESS" (HIPER = HIgh PERformance) ${ }^{*}$.

The world-wide release of an adequate unlicensed spectrum for personal communication systems at $5 \mathrm{GHz}$ (much more bandwidth is available at higher frequencies bands around 40 and $60 \mathrm{GHz}$ ) is an import prerequisite for the realization of local area multimedia radio networks. For broadband radio access networks appropriate frequency bands are available region-dependent at $3.5,10,24 / 26,28 \mathrm{GHz}$, and further bandwidth above $30 \mathrm{GHz}$ is under discussion.

The paper presents the protocol stack for the air interface of an ATM fixed radio access network with directional antennas at terminals and sectored antennas at base stations (also called Point-to-Multipoint system). The system is intended mainly for the access of small and medium business users. The basic parameters of the system are summarized in Table 1 . A radio access network usually contains multiple base stations each with several radio sectors (Figure 1). A feeder network connects the base stations to the line termination with the VB5 interface. It is assumed that interference between radio channels of neighbouring sectors is avoided by using orthogonal radio resources which are divided by directional antennas, polarization and frequency. Aspects of the physical layer (modem, antennas, etc.) as well as the networks behind the air interface are outside the scope of the paper.

The W-ATM system has to fit transparently into a fixed ATM network. Therefore, the protocol stack at the air interface has to execute statistical multiplexing of ATM

"Furthermore, a "HIPERLINK" standard for short distance ATM radio relay systems in an unlicensed frequency band is planed. 

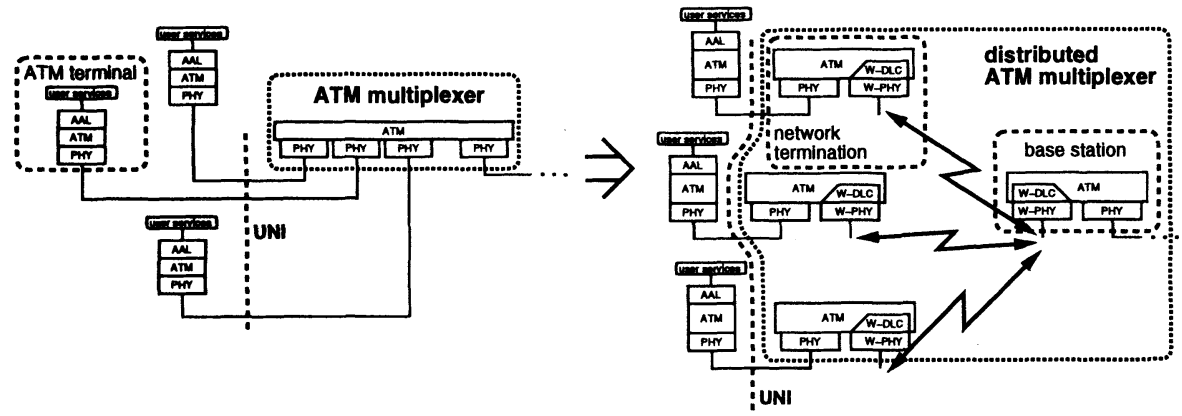

Figure 2 Correspondence between radio sector and ATM multiplexer

cells as in fixed ATM multiplexers. A medium access control (MAC) protocol is required to coordinate the competition of terminals for the shared radio channel.

The approach for the MAC protocol presented here takes into account that the performance of ATM networks is mainly influenced by the intelligence of the ATM cell multiplexing in ATM network nodes. Modern ATM multiplexers apply service strategies that optimize the resource allocation based on short-term demands of virtual channels and their negotiated quality of service (QoS). The ATM air interface can be interpreted as a distributed ATM multiplexer. The MAC protocol is centrally controlled by the base station and realizes the transmission order of ATM cells given by the service strategy.

The paper has the following structure. Section 2 introduces the architecture and protocol stack of the ATM air interface. In section 3 the service strategy for the air interface is specified. Section 4 describes how the distributed ATM cell scheduler is realized. The MAC protocol is described in section 5. Section 6 presents a random access protocol for the fast and efficient signaling of capacity requests over the uplink. The paper winds up with a conclusion.

\section{ARCHITECTURE OF AN ATM AIR INTERFACE}

In general, the users at a W-ATM access network request the same functionality and QoS as users of wired ATM access networks. Figure 2 illustrates how these user requirements can be transformed into the demand on building a distributed ATM multiplexer around the air interface which is characterized by a radio channel inside. The figure only considers the user plane of the multiplexer. The wireless terminal is equivalent to the network termination (W-NT) of the access network and offers one or multiple User-Network interfaces (UNI) to connect customer premises equipment (see also Figure 1).

At the air interface additional layers must be introduced in the protocol stack. The resulting stack contains a wireless physical layer with the radio modems below the ATM layer and a data link control (DLC) layer that contains the ATM cell scheduler. 


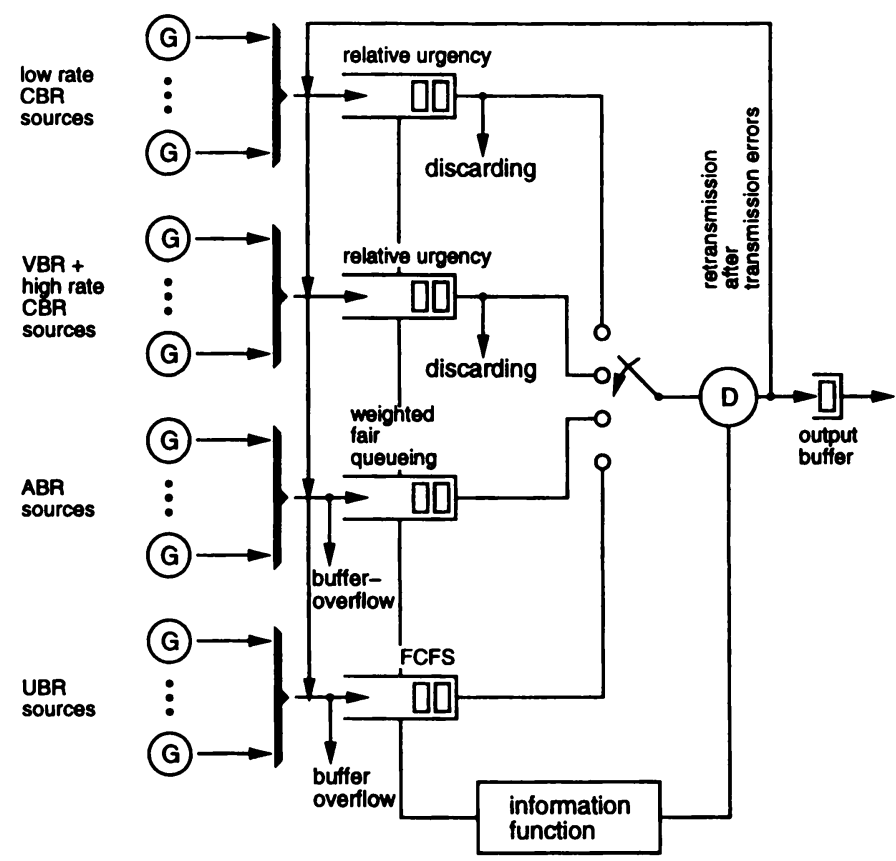

Figure 3 Queueing model for the upstream of the distributed ATM multiplexer

The DLC layer is furthermore divided into a MAC sublayer and a Logical Link Control (LLC) sublayer.

\section{SERVICE STRATEGY AT THE AIR INTERFACE}

Network nodes (switches and multiplexers) have to determine the transmission order of cells to be sent over a link. An ATM cell scheduler is introduced for each output port/link that controls the statistical multiplexing of cells by employing an appropriate service strategy. Its goal is to optimize the resource allocation based on short-term demands of virtual channels and their negotiated quality of service. Appropriate service strategies usually focus on two key targets: avoiding overflow of buffers and controlling delays of ATM cells. Which issue the strategy has to focus on strongly depends on the transfer rate of the link. While a fast transfer rate of 155 Mbit/s and more in wired ATM networks causes buffer overflows to be the more critical aspect, with slow transfer rates, e.g. $8 \mathrm{Mbit} / \mathrm{s}(\approx 20.000 \mathrm{cells} / \mathrm{s})$ on the upstream in W-ATM systems, cell delay guarantees become more difficult to fulfill and the service strategy plays a major role in providing QoS.

A service policy has to distinguish between the real-time oriented CBR/VBR services and non-real-time ABR/UBR services. As in most fixed ATM multiplexers, static priorities are introduced between service categories (CBR/VBR $>$ ABR $>$ UBR). For the upstream another high priority level is introduced for low rate CBR 


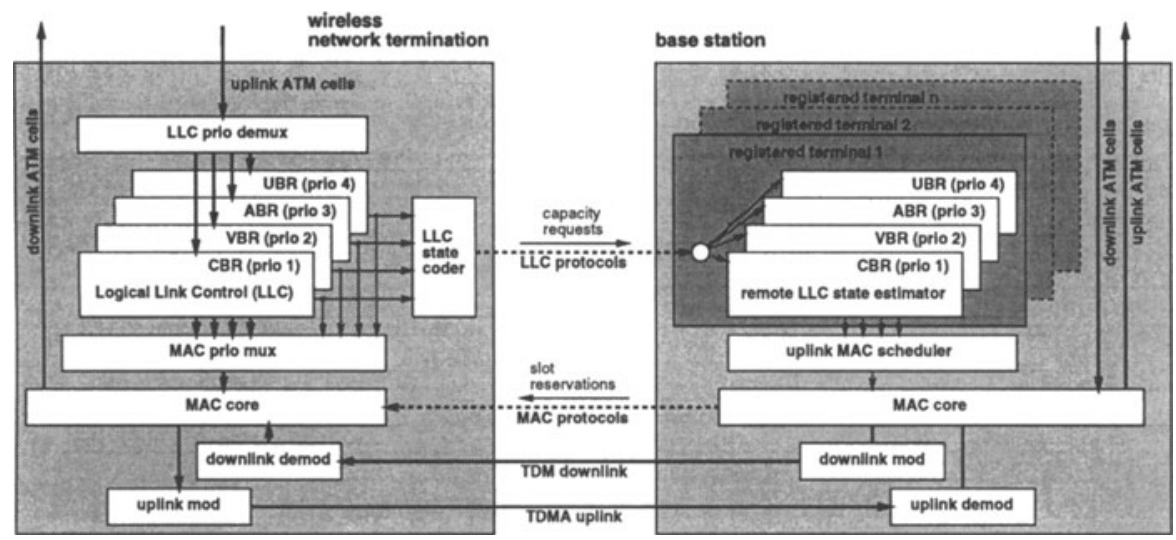

Figure 4 Dividing the upstream scheduler into an LLC and MAC part

connections ( $\leq 128 \mathrm{kbit} / \mathrm{s})$ in order to prevent voice services from congestion events caused by bursty VBR connections (Figure 3). For ABR services the algorithms applied for fixed ATM multiplexers are used (e.g., weighted fair queueing (COST 242 Management Committee, 1996)), while the UBR service category requires fair resource sharing.

For real-time oriented CBR and VBR services a specific service strategy has to controls delays. The connection specific performance parameter maximum cell transfer delay $(\max C T D)$ is specified by the traffic contract and is related to the end-toend delay. The air interface as one hop of a virtual channel connection is only allowed to consume a fraction of $\max C T D$. A maximum delay $\tau_{d \max }$ on the air interface is not specified. The assumption of $\tau_{d \max }=0.1 \max C T D$ seems to be useful. A detailed analysis of appropriate service strategies for real-time services can be found in (Kist and Petras, 1997). The reference recommends dynamic priorities where the priorities of ATM cells depend on their waiting time and their connection specific QoS requirements. An arriving cell gets a due date by adding $\tau_{d \max }$ to its arrival time. Accourding to the strategy the cell with earliest due date (EDD) is transmitted first. If all connections are considered to have the same maximum delay, the EDD strategy is equivalent to the First Come First Serve (FCFS) strategy. The more complex Relative Urgency (RU) discipline furthermore considers the maximum cell loss ratio and minimizes the probability for cells being late (exceeding their due dates). ATM cells which exceed their maximum delay will usually be discarded by the receiving application. Thus, discarding delayed cells (due date expired) at the air interface contributes to avoid and resolve congestion events, since the delay of the following cells can be shortened and the probability to exceed further due dates is reduced. 


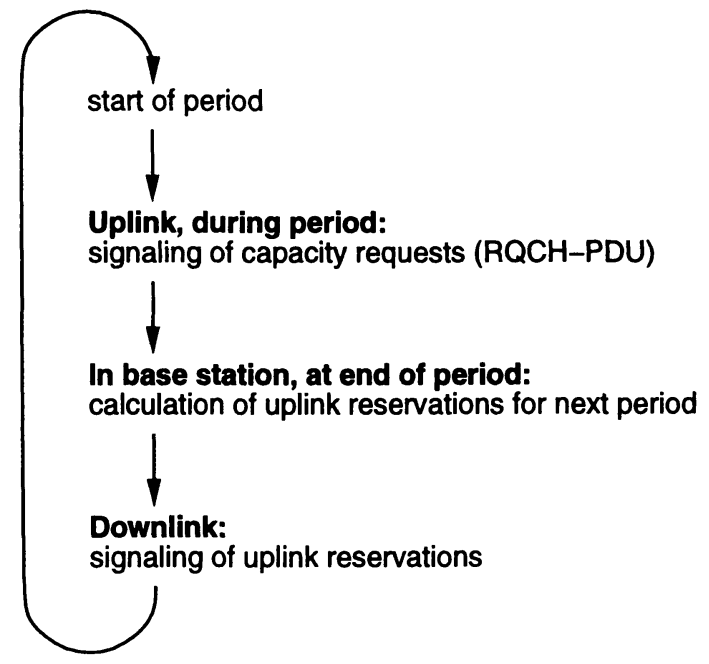

Figure 5 Cycle of signaling periods in DSA++ MAC protocol

\section{REALIZING THE DISTRIBUTED ATM CELL SCHEDULER}

The main difference between the distributed ATM multiplexer and a fixed ATM multiplexer is the distribution of the ATM cell scheduler for upstream cells between WNT and the base station. For the downstream, the scheduler is completely contained in the DLC layer of the base station.

For the uplink the ATM cell scheduler is separated into two parts. The lower part in the MAC sublayer selects the W-NT which is allowed to send in a time slot (determination of slot reservations). The upper part of the scheduler belonging to the LLC sublayer contains the send buffers and determines a virtual channel for delivering ATM cells which are transmitted in a reserved time slot.

The LLC sublayer in W-NTs contains an entity for each priority level of the service strategy with the send buffers of the corresponding service category. The base station contains mirrors of the W-NT entities each estimating the state of the send buffers in the corresponding W-NT entity. A signaling protocol is executed for notifying the mirror entities about the states of the W-NT entities. This is done by generating (in the W-NT) and interpreting (in the base station) capacity request messages, which are transmitted as RQCH-PDUs (ReQuest CHannel Protocol Data Unit) by the RQCH service of the MAC sublayer (cf. section 6).

The MAC scheduler uses the capacity requests of W-NTs, which are estimated by the mirror instances, in order to determine the reservation of upstream slots. The MAC core performs a signaling protocol (cf. section 5) that notifies the W-NTs about the slot reservations.

Furthermore, the MAC core is responsible for the transmission of RQCH-PDUs. 


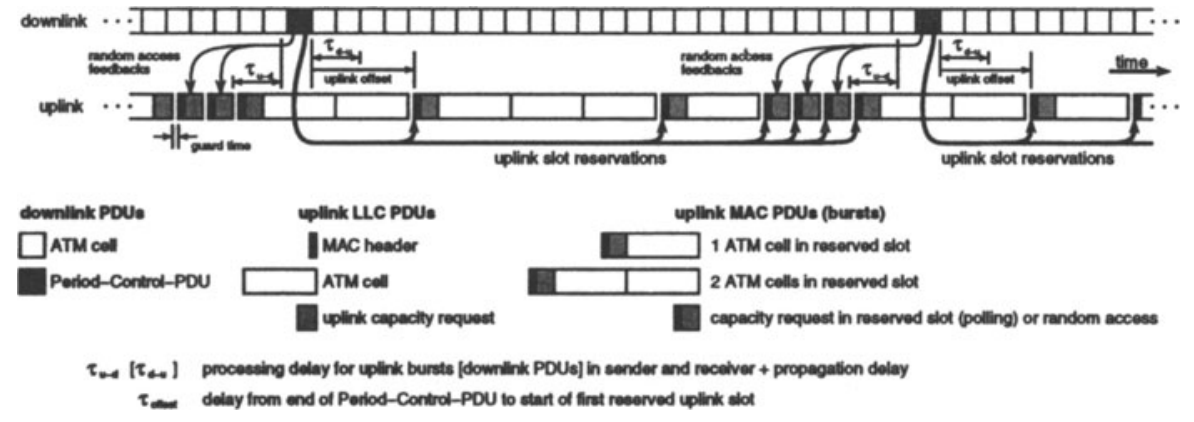

Figure 6 Downlink signaling scheme of DSA++ protocol

\section{MEDIUM ACCESS CONTROL PROTOCOL}

Active W-NT have to register at the base station. A temporary MAC identifier (MACId, 8 bit) is assigned to each registered W-NT, which is used as a short address within the sector for addressing uplink slot reservations.

On the downlink ATM cells are broadcasted and received by all W-NTs (Figure 6). The virtual path identifier (VPI) is used to detect the target W-NT. MAC-internal signaling messages are carried by ATM cells with a specific mark, e.g., VPI $=0$ or OAM F3. On the uplink the VPI may also be used to indicate the destination W-NT of ATM cells. For safety reasons, each uplink burst is extended by a MAC header containing the MAC-Id and a short identification of the sector to enable the detection of faulty insertions of bursts received from neighbouring sectors.

The MAC protocol executed by the MAC core is called Dynamic Slot Reservation (DSA++) protocol and has originally been developed for HIPERLAN type 2 systems on a time division duplex (TDD) link (Petras and Krämling, 1997b). For broadband radio access networks it has been modified in order to work on a frequency division duplex (FDD) link with asymmetric link rates.

Uplink slots allow the transmission of one physical burst that contains an information field of variable length together with the necessary overhead of the physical layer (synchronization of terminals to balance propagation delays is assumed). The information field carries one RQCH-PDU and up to four ATM cells. Slots which allow the transmission of a burst with only an RQCH-PDU and no ATM cell are called RQCH slots or short slots.

In order to co-ordinate the channel access, the DSA++ MAC protocol groups uplink slots in so-called signaling periods. The periodic signaling procedure is illustrated in Figure 5. A signaling period consists of a variable number of uplink slots, the number and order of which is determined by the MAC scheduler.

During such a signaling period new capacity requests arrive at the base station and are saved in the mirror entities. At the end of a period the slot reservations of the next period are calculated and transmitted to the W-NTs. This is done with a PeriodControl-PDU on the downlink that signals the number of slots in the next signaling period and for each slot its length (number of ATM cells) and the associated MAC- 


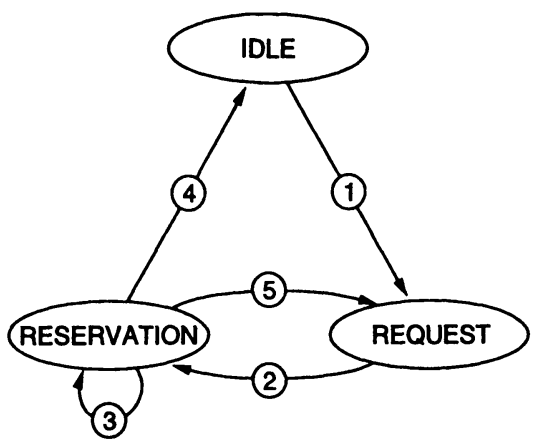

(1) arrival of burst of ATM cells

(2) successful transmission of capacity request message (RQCH-PDU)

(3) piggyback transmission of $\mathrm{RQCH}-$ PDU

(4) transmission of last ATM cell from burst, empty send buffer

(5) arrival of ATM cell of service category with higher priority

Figure 7 Reservation states of W-NT

Id. A signaling period may contain a variable number of short slots for RQCH-PDUs (Figure 6). The access to the RQCH slots is performed with random access under control of the RQCH protocol explained in section 6.

The Period-Control-PDU furthermore contains a feedback (MAC acknowledgment) for each random access slots of the last signaling period which consists of the MAC-Id of the received bursts. The feedback is used by the RQCH access protocol to resolve collisions. By also sending a feedback for each ATM cell, erroneous transmission of ATM cells can be detected and retransmissions be triggered. Such an error control protocol is for further study.

The maximum length of a signaling period is given by the limited size of the Period-Control-PDU carrying the slot reservations. Furthermore, too long signaling periods reduce the dynamics of the protocol and cause too long delays for real-time services. Experience has shown that a signaling period should not exceed $0.5 \mathrm{~ms}$ (Petras and Krämling, 1997b).

\section{SIGNALING OF CAPACITY REQUESTS OVER THE UPLINK}

Capacity requests (RQCH-PDU) are transmitted depending on the reservation state of a W-NT. The diagram in Figure 7 shows the corresponding state transition diagram. State IDLE corresponds to an empty send buffer so that no capacity request message is to be sent. After the arrival of an ATM cell the W-NT switches to state REQUEST (transition (1)) and tries to transmit its capacity request in a short slot. After a successful transmission the W-NT enters state RESERVATION (transition (2)) and will be served by the scheduler according the urgency of its ATM cells. With the transmission of an ATM cell in a reserved long slot the scheduler is informed about the newest capacity requirements by means of the piggybacked RQCH-PDU (transition (3)). If no further capacity is required, the base station recognizes that the W-NT has returned to state IDLE (transition (4)).

A special case is the parallel existence of virtual channels of different service categories. If a W-NT did request capacity for a low priority service category (e.g. UBR) and thus is in state RESERVATION, than the arrival of an ATM cell of a high 


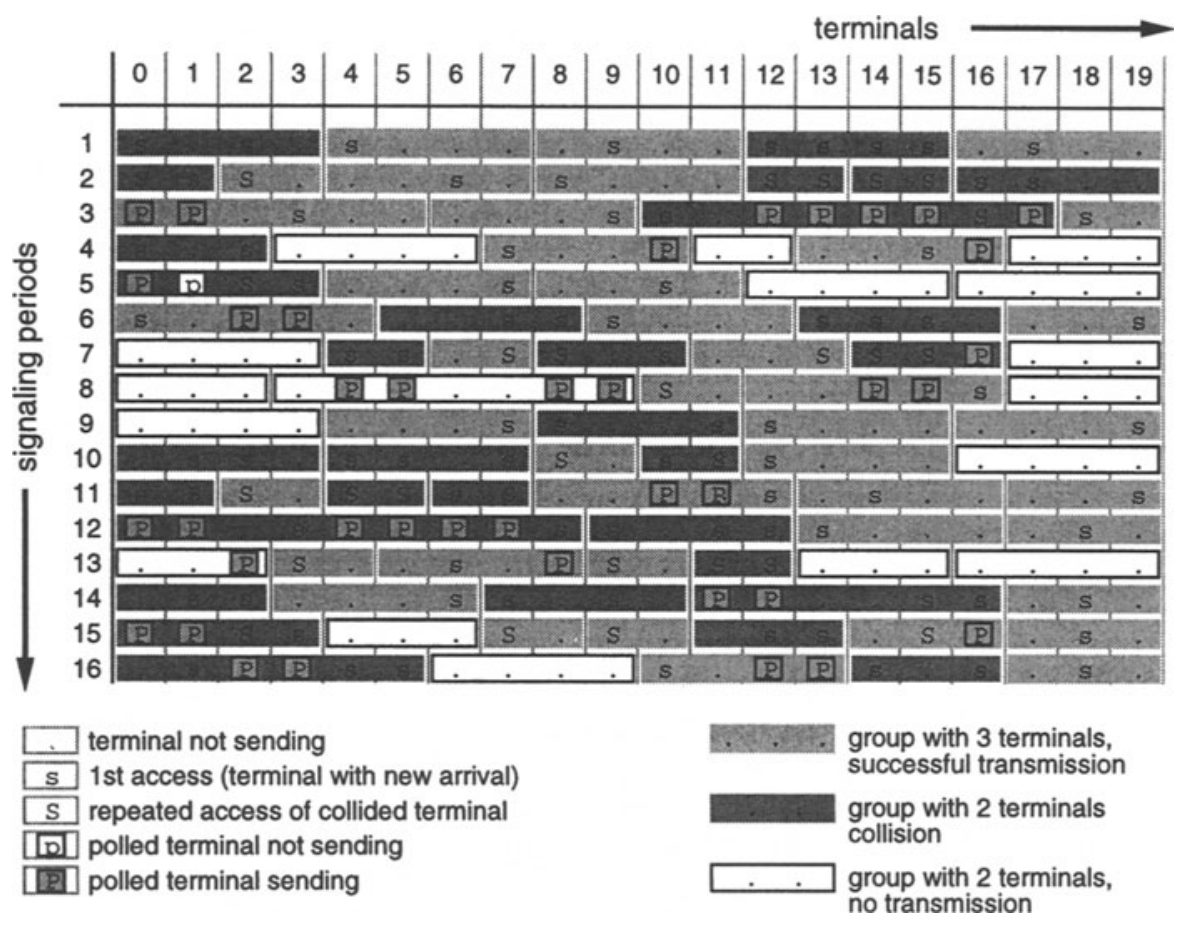

Figure 8 Example protocol sequence of probing algorithm over 16 signaling periods with 20 registered W-NT

priority service category (e.g. VBR) may modify the capacity requirements in such a way, that the W-NT is not able to wait for the next reserved slot in order to piggyback the newest capacity request on ATM cells. Instead it goes back to state REQUEST (transition (5)) and forces the retransmission of its capacity request over the RQCH.

Due to the urgency of the transmission of capacity requests, the random access of the RQCH protocol must not be optimized for throughput but for short delays. A critical item are the delayed feedbacks, because a second random access of the same W-NT is only useful, if the feedback of the first access has been evaluated before. Therefore, dedicated shortened signaling periods are useful to enable fast transmission of feedbacks.

The proposed collision resolution algorithm called probing algorithm (Petras and Krämling, 1997a; Petras and Krämling, 1997b) is a non blocking adaptive identifier splitting algorithm that takes advantage of the known number of stations in contention mode, since only registered W-NTs are allowed to request capacity. The algorithm combines the advantages of random access and polling. Thus, it is able to keep access delays below a predefined W-NT specific limit, as it is required for the support of real-time multimedia services in ATM networks.

At the beginning of each signaling period the probing algorithm divides the identifier space in a variable number $t$ of consecutive intervals and assigns one short slot 
to each interval. The Period-Control-PDU signals the interval division to the W-NTs by transmitting the start identifier of each interval. Furthermore, it is possible to poll specific W-NTs in dedicated short slots. Such W-NTs are not allowed to send in random access slots.

The width of each interval is determined by considering the probability $p_{s e n d, i}$ that W-NT $i$ will send in a random access slot. After each access $p_{s e n d, i}$ is corrected taking into account the knowledge gathered from the results of the last access. The example in Figure 8 illustrates how intervals with collided terminals are split into smaller intervals in order to resolve the collision (accourding to identifier splitting). Details about the algorithms for estimating and correcting $p_{s e n d, i}$ are outside the scope of this paper but can be found in (Petras and Krämling, 1997a).

\section{CONCLUSIONS}

The protocol stack for a fixed ATM radio access network has been presented. The development of the protocol stack was based on the objective to implement an distributed ATM multiplexer with an ATM cell scheduler as in modern fixed ATM network nodes. Therefore, it is able to transparently extend ATM over a Pointto-Multipoint radio channel. Although wireless ATM systems with mobility like HIPERLAN type 2 are still under research (e.g., dynamic channel allocation in unlicensed frequency bands, handover due to mobility, error control on a mobile radio channel), the protocols for fixed W-ATM systems are well investigated. Bosch Telecom currently executes development activities to extend its narrowband Point-toMultipoint product (Digital Multipoint System, DMS) by broadband ATM services.

\section{REFERENCES}

Agrawal, P., Hyden, E., Krzyzanowski, P., Mishra, P., Srivastava, M. and Trotter, J. (1996). SWAN: A Mobile Multimedia Wireless Network, IEEE Personal Communications Magazine 3(2): 18 - 33.

Ala-Laurila, J. and Awater, G. (1997). The Magic WAND - Wireless ATM Network Demonstrator System, ACTS Mobile Summit '97, Aalborg, Denmark.

BRAN Project (1997). Broadband Radio Access Networks (BRAN): Terms of References, Technical report, ETSI.

Ciotti, C. (1996). The AC006 MEDIAN Project, an Overview and State of the Art, ACTS Mobile Communications Summit.

COST 242 Management Committee (1996). Methods for the performance evaluation and design of broadband multiservice networks, Part I Traffic control, Final Report Seminar version, COST.

DAVIC (1997). DAVIC 1.3 Specification Part 8: Lower Layer Protocols and Physical Interfaces, Technical Report Rev. 6.2, DAVIC.

Dinis, M., Lagarto, V., Prögler, M. and Zubrzycki, J. (1997). SAMBA - A Step to bring MBS to the People, ACTS Mobile Summit '97, pp. 495-500. 
Eng, K., Karol, M., Veeraraghavan, M., Ayanoglu, E., Woodworth, C., Pancha, P. and Valenzuela, R. (1995). A wireless broadband ad-hoc ATM local area network, Wireless Networks (1): 161-174.

Kist, H. and Petras, D. (1997). Service Strategy for VBR Services at an ATM Air Interface, EPMCC'97, Bonn, Germany.

Petras, D. and Krämling, A. (1997a). Fast Collision Resolution in Wireless ATM Networks, 2nd MATHMOD, Vienna, Austria.

Petras, D. and Krämling, A. (1997b). Wireless ATM: Performance Evaluation of a DSA++ MAC Protocol with Fast Collision Resolution by a Probing Algorithm, Int. J. of Wireless Information Networks 4(4).

Porter, J. and Hopper, A. (1995). An Overview of the ORL Wireless ATM System, IEEE ATM Workshop, Washington, DC, pp. 18 - 33 .

Radimirsch, M. (1997). ATMmobil Overview, ACTS Wireless-ATM Workshop 1997, Brussels, Belgium.

Raychaudhuri, D., French, L., Siracusa, R., Biswas, S., Yuan, R., Narasimhan, P. and Johnston, C. (1997). WATMnet: A Prototype Wireless ATM System for Multimedia Personal Communication, IEEE J. Selected Areas in Communications 15(1): 83-95.

Walke, B., Petras, D. and Plassmann, D. (1996). Wireless ATM: Air Interface and Network Protocols of the Mobile Broadband System, IEEE Personal Communications Magazine 3(4): 50-56.

Wireless ATM Group (1997). Baseline Text for Wireless ATM Specifications, Technical report, ATM Forum. 